\title{
DETERMINATION OF THE TYPE AND PROPERTIES OF THE MAPPING FUNCTION OF A CLASS OF DOUBLY- CONNECTED RIEMANN SURFACES
}

\author{
HOWARD E. TAYLOR
}

1. Introduction. ${ }^{1}$ The object of this paper is to consider a class of open doubly-connected Riemann surfaces ${ }^{2}$ and to show that the members of the class are images of the complex plane less two points; also a representation of the mapping function is obtained. To this end a class of open simply-connected Riemann surfaces is defined and the members shown to be parabolic and a representation of the mapping function is obtained.

The methods employed involve approximation by sequences of elliptic surfaces developed by G. R. MacLane $[8]^{3}$ and the use of the results of Carathéodory [2] on the mapping of a sequence of plane domains by a family of functions.

In the terminology of Iversen [7] certain members of each class of surfaces exhibit indirectly critical singularities.

2. Description of the class of simply-connected surfaces. A surface $\mathcal{Z}$ of this class is defined by two infinite sequences of real numbers $\left\{a_{n}\right\},\left\{b_{n}\right\}(n=1,2, \cdots)$ with $0<a_{1}<b_{1}, a_{2 k \pm 1}>a_{2 k}, b_{2 k-1}>a_{2 k-1}$, $a_{2 k}>b_{2 k}$. $\mathcal{F}$ consists of sheets $S_{1}, S_{2}, \cdots, S_{k}, \cdots$, each sheet being a slit copy of the $w$-sphere. $S_{1}$ is cut along the positive real axis from $w=a_{1}$ to $w=b_{1} . S_{k}(k>1)$ is cut along the real axis from $a_{k-1}$ to $b_{k-1}$ and from $a_{k}$ to $b_{k}$. $S_{1}$ and $S_{2}$ are joined along their cuts from $a_{1}$ to $b_{1}$ forming first order branch points over $a_{1}$ and $b_{1} . S_{2 k}$ and $S_{2 k+1}$ are joined along their cuts from $a_{2 k}$ to $b_{2 k}, S_{2 k}$ and $S_{2 k-1}$ are joined along their cuts from $a_{2 k-1}$ to $b_{2 k-1}$ forming first order branch points over $w=a_{n}$ and $w=b_{n}, n=1,2,3, \cdots . \mathcal{F}$ is topographically equivalent to a semi-infinite cylinder, hence is open and simply-connected. Therefore $\mathcal{F}$ is either hyperbolic or parabolic.

The nature of the singularities of $\mathcal{F}$ depends on the sequences $\left\{a_{n}\right\},\left\{b_{n}\right\}$. (1) If neither sequence has a limit, $\mathcal{F}$ has no singularities. (2) If one of the sequences has a limit or if both have the same limit,

Presented to the Society, December 28, 1950; received by the editors April 24, 1952.

1 This paper is an extract from a thesis presented to the Faculty of the Rice Institute in June, 1950.

2 The author is greatly indebted to Professor Gerald R. MacLane for calling his attention to this problem and for his friendly encouragement and valuable counsel.

${ }^{3}$ Numbers in brackets refer to the references listed at the end of the paper. 
$\mathcal{F}$ has one indirectly critical singularity. (3) If $a_{n} \rightarrow a \neq \infty$ and $b_{n} \rightarrow \infty$, $\mathcal{F}$ has two indirectly critical singularities.

3. Description of the class of doubly-connected surfaces. A surface $\mathcal{F}$ of this class is defined by two infinite sequences of real numbers $\left\{a_{n}\right\},\left\{b_{n}\right\}, n= \pm 1, \pm 2, \pm 3, \cdots$, with $b_{-1}<a_{-1}<0<a_{1}<b_{1}, a_{2 \pm \pm 1}$ $>a_{2 k}, \quad b_{2 k-1}>a_{2 k-1}, \quad a_{2 k}>b_{2 k}, a_{-2 k}>a_{-2 k \pm 1}, a_{-2 k-1}>b_{-2 k-1}, \quad b_{-2 k}>a_{-2 k}$, $k \geqq 1$. $\mathcal{F}$ consists of an infinite number of sheets

$$
\begin{array}{r}
\cdots, S_{-k}, S_{-k+1}, \cdots, S_{-1}, S_{1}, S_{2}, \cdots, S_{k}, S_{k+1}, \cdots, \\
k=1,2,3, \cdots,
\end{array}
$$

each sheet being a slit copy of the $w$-sphere. $S_{j}, j=-1, \pm 2, \pm 3, \cdots$, is cut along the real axis from $a_{j}$ to $b_{j}$ and from $a_{j-1}$ to $b_{j-1} . S_{1}$ is cut from $a_{1}$ to $b_{1}$ and from $a_{-1}$ to $b_{-1} . S_{j}$ and $S_{j+1}$ are joined along their cuts between $a_{j}$ and $b_{j}$ for $j \neq-1 . S_{-1}$ and $S_{1}$ are joined along their cuts between $a_{-1}$ and $b_{-1}$. Branch points of first order are formed over $w=a_{j}$ and $w=b_{j}, j= \pm 1, \pm 2, \pm 3, \cdots . \mathcal{F}$ is topologically equivalent to an infinite cylinder, hence is open and doubly-connected. By the uniformizing principle [6], $\mathcal{F}$ can be mapped onto the $\zeta$-plane slit along two line segments parallel to the real axis and thence onto the annulus $0 \leqq r<|z|<R \leqq \infty$ in the $z$-plane [3, pp. 71-72].

$\mathcal{F}$ can have no, one, two, three, or four singularities. For consider $\mathcal{F}$ as made up of two surfaces $\mathcal{F}_{1}$ and $\mathcal{F}_{2}$ where $\mathcal{F}_{1}$ consists of sheets of positive subscript and $\mathcal{F}_{2}$ of sheets of negative subscript, and the singularities of $\mathcal{F}_{1}$ and $\mathcal{F}_{2}$ can be classified as in $\$ 2$. So any singularities of $\mathcal{F}$ will be indirectly critical.

4. Proof that all surfaces of the first class are parabolic. Let $\mathcal{F}$, a member of the class of simply-connected surfaces, be mapped onto the circle $|z|<R \leqq \infty$ by the function

$$
z=\phi(w), \quad w=f(z), \quad f(0)=0 \in S_{1}, \quad f^{\prime}(0)=1 .
$$

To determine the images in the z-plane of the branch points of $\mathcal{F}$, consider the two symmetric halves of $\mathcal{F}$ obtained by slicing each sheet along the uncut portion of the real axis (i.e., $S_{k}$ is sliced from $b_{k}$ to $(-1)^{k+1} \infty$, from $b_{k-1}$ to $(-1)^{k} \infty$, and from $a_{k}$ to $\left.a_{k-1}\right)$. The half of $\mathcal{f}$ containing the upper half of $S_{1}$ can be mapped on a semi-circle $|z|$ $<R \leqq \infty, \Im(z)>0$, so that the point over the origin in $S_{1}$ is mapped onto $z=0$, the point over $\infty$ in $S_{1}$ is mapped onto $z=\gamma_{1}<0$, the edges of the slices from $-\infty$ to $a_{1}$ and from $a_{k-1}$ to $a_{k}$ are mapped on the diameter $-R<z<R$ to the right of $\gamma_{1}$, and the edges of the slices from 
$b_{k}$ to $(-1)^{k+1} \infty$ are mapped on the diameter to the left of $\gamma_{1}$. This mapping carries the branch points over $\left\{a_{n}\right\}$ into a monotone increasing sequence of points $\left\{\alpha_{n}\right\}$ on the positive real axis and the branch points over $\left\{b_{n}\right\}$ into a monotone decreasing sequence of points $\left\{\beta_{n}\right\}$ on the negative real axis of the $z$-plane. The point over $\infty$ in $S_{k}$ is carried into the point $\gamma_{k}$ on the real axis with $\beta_{k}<\gamma_{k}<\beta_{k-1}$. If we apply the Schwartz reflection principle to the inverse of this mapping function and normalize, we are led to the function (1), so $f(z)$ is real for $z$ real and the image of the branch point of $\mathcal{F}$ over $a_{k}$ is $z=\alpha_{k}$ with $0<\alpha_{1}<\alpha_{2}<\cdots<\alpha_{k}<\alpha_{k+1}<\cdots$ and the image of the branch point of $\mathcal{F}$ over $b_{k}$ is $z=\beta_{k}$ with $0>\beta_{1}>\beta_{2}>\cdots>\beta_{k}$ $>\beta_{k+1}>\cdots$ and the image of the point of $\mathcal{F}$ in $S_{k}$ over $w=\infty$ is $z=\gamma_{k}$ with $0>\gamma_{1}>\beta_{1}>\gamma_{2}>\beta_{2}>\cdots>\beta_{k-1}>\gamma_{k}>\beta_{k}>\cdots S_{1}$ is mapped on a portion of $|z|<R$ bounded by a simple closed curve $C_{1}$, symmetric about the real axis and cutting the real axis in $\alpha_{1}$ and $\beta_{1}$ only. $S_{k}(k>1)$ is mapped on a portion of $|z|<R$ bounded by two nonintersecting, simple, closed curves $C_{k-1}$ and $C_{k}$, each symmetric about the real axis with $C_{k-1}$ cutting the real axis at $\alpha_{k-1}$ and $\beta_{k-1}$ only, and with $C_{k}$ cutting the real axis at $\alpha_{k}$ and $\beta_{k}$ only. The uncut segment of the real axis of $S_{1}$ corresponds to the segment $\left(\beta_{1}, \alpha_{1}\right)$ and the two shores of the cut from $a_{1}$ to $b_{1}$ correspond to the two symmetric halves of $C_{1}$. The uncut segment $\left(a_{k-1}, a_{k}\right)$ of $S_{k}(k>1)$ corresponds to the segment $\left(\alpha_{k-1}, \alpha_{k}\right)$ and the remaining uncut portion of the real axis of $S_{k}$ corresponds to the segment $\left(\beta_{k-1}, \beta_{k}\right)$. The shores of the cut $\left(a_{k-1}, b_{k-1}\right)$ correspond to the two symmetric halves of $C_{k-1}$ and the shores of the cut $\left(a_{k}, b_{k}\right)$ correspond to the two symmetric halves of $C_{k}$. The curves $C_{k}$ and the real axis are the paths on which $w=f(z)$ is real.

Consider the elliptic surface $\mathcal{F}_{n}$ which consists of the first $n+1$ sheets of $\mathcal{F}$ with the cut from $a_{n+1}$ to $b_{n+1}$ healed. $\mathcal{F}_{n}$ is a simply-connected closed surface with $2 n$ first order branch points over $a_{1}, a_{2}, \cdots, a_{n}, b_{1}, b_{2}, \cdots, b_{n}$ and with $n+1$ points over $w=\infty$. Hence $\mathcal{F}_{n}$ is the Riemann surface of the inverse of a rational function $w=R_{n}(z)$ which can be normalized so that $R_{n}(0)=0 \in S_{1}, R_{n}^{\prime}(0)=1$, $R_{n}(\infty)=\infty \in S_{n+1}$. $R_{n}(z)$ has $n+1$ simple poles, $n$ of them at the images $\boldsymbol{\gamma}_{1, n}, \boldsymbol{\gamma}_{2, n}, \boldsymbol{\gamma}_{3, n}, \cdots, \boldsymbol{\gamma}_{n, n}$ of the points over $w=\infty$ in the sheets $S_{1}, \cdots, S_{n}$ and one at $z=\infty$, and $R_{n}^{\prime}(z)$ must have $2 n$ first order zeros at the images $\alpha_{1, n}, \alpha_{2, n}, \cdots, \alpha_{n, n}, \beta_{1, n}, \beta_{2, n}, \cdots, \beta_{n, n}$ of $a_{1}, \cdots, a_{n}, b_{1}, \cdots, b_{n}$ and no other zeros, where $\beta_{n, n}<\gamma_{n, n}<\beta_{n-1, n}$ $<\cdots<\beta_{1, n}<\gamma_{1, n}<0<\alpha_{1, n}<\alpha_{2, n}<\cdots<\alpha_{n, n}$. Hence $R_{n}(z)$ $=P_{n}(z) / Q_{n}(z)$ where $P_{n}$ is a polynomial of degree $n+1$ and $Q_{n}$ is a polynomial of degree $n$, and we can write $R_{n}(z)=\int_{0}^{z} R_{n}^{\prime}(t) d t$, 
$R_{n}^{\prime}(z)=\prod_{k=1}^{n}\left(1-z / \alpha_{k, n}\right)\left(1-z / \beta_{k, n}\right) /\left(1-z / \gamma_{k, n}\right)^{2}$ where the residues of $R_{n}^{\prime}(z)$ at the poles are zero.

Let $D_{n}$ be the $z$-plane cut from $\alpha_{n, n}$ to $\infty$ along the positive real axis. $D_{n}$ is mapped by $w=R_{n}(z)$ onto $\mathcal{F}_{n}$ with the sheet $S_{n+1}$ cut from $a_{n}$ to $(-1)^{n} \infty$ along the real axis. But $\zeta=\phi(w)$ maps this cut surface one-to-one on the domain $\Delta_{n}$ of the $\zeta$-plane bounded by the curve $C_{n+1}$ and the segments $\left(\beta_{n+1}, \gamma_{n+1}\right),\left(\alpha_{n}, \alpha_{n+1}\right)$ and containing $\zeta=0$. So $\zeta=\phi\left[R_{n}(z)\right]=\psi_{n}(z)$ provides a schlicht mapping of $D_{n}$ onto $\Delta_{n}$ with $\psi_{n}(0)=0$ and $\psi_{n}^{\prime}(0)=1$. The following is an immediate corollary of Koebe's distortion theorem: Let $w=f(z)$ be holomorphic in the $z$-plane slit from $z=R>0$ to $+\infty$ along the real axis and map this schlitzbereich on a plane domain $\Delta$ of the $w$-plane subject to the conditions $f(0)=0, f^{\prime}(0)=1$. Then the distance from $w=0$ to the boundary of $\Delta$ is greater than or equal to $R$. Applying this, we have that the distance from $\zeta=0$ to the curve $C_{n+1}$ is greater than $\alpha_{n, n}$.

For $0<z<\alpha_{1, n}, \prod_{k=1}^{n}\left(1-z / \beta_{k, n}\right) /\left(1-z / \gamma_{k, n}\right)<1, \prod_{k=1}^{n}\left(1-z / \gamma_{k, n}\right)$ $>1, \prod_{k=1}^{n}\left(1-z / \alpha_{k, n}\right)>0,0<R_{n}^{\prime}(z)<\prod_{k=1}^{n}\left(1-z / \alpha_{k, n}\right)$, and if $1 / \bar{\alpha}_{n}$ $=(1 / n) \sum_{k=1}^{n} 1 / \alpha_{k, n}, R_{n}^{\prime}(z)<\left[(1 / n) \sum_{k=1}^{n}\left(1-z / \alpha_{k, n}\right)\right]^{n}=\left(1-z / \bar{\alpha}_{n}\right)^{n}$. So $a_{1}=\int_{0}^{\alpha_{1, n}} R_{n}^{\prime}(z) d z<\int_{0}^{\alpha_{1, n}}\left(1-z / \bar{\alpha}_{n}\right)^{n} d z<\int_{0}^{\bar{\alpha}_{n}}\left(1-z / \bar{\alpha}_{n}\right)^{n} d z=\bar{\alpha}_{n} /(n+1)$, and since $\sum_{k=1}^{n} 1 / \alpha_{k, n}<n /(n+1) a_{1}<1 / a_{1}$ we have for any $\nu, 1 \leqq \nu \leqq n$, $\nu / \alpha_{\nu, n}<\sum_{k=1}^{n} 1 / \alpha_{k, n}<1 / a_{1}$, or $\alpha_{\nu, n}>a_{1} \nu$ for $\nu=1,2, \cdots, n ; n$ $=1,2, \cdots$. Therefore the distance from the origin to $C_{n+1}$ is greater than $a_{1} n$ for all $n$, and $\mathcal{F}$ is parabolic.

5. Structure of the mapping function for surfaces of the first class. We have shown above that $D_{n}$ converges to $|z|<\infty$ and $\Delta_{n}$ converges to $|\zeta|<\infty$, and using the form of Carathéodory's theorem [2, pp. 118-126] on families of schlicht mappings which is stated in Bieberbach $\left[1\right.$, p. 13], we conclude: $\phi\left[R_{n}(z)\right] \rightarrow z$ uniformly in $|z| \leqq r$ for any finite $r$ and $R_{n}(z) \rightarrow \phi^{-1}(z)=f(z)$ uniformly in $|z| \leqq r$. By Hurwitz' theorem [5, p. 249], $\lim _{n \rightarrow \infty} \alpha_{k, n}=\alpha_{k}, \lim _{n \rightarrow \infty} \beta_{k, n}=\beta_{k}, \lim _{n \rightarrow \infty} \gamma_{k, n}$ $=\gamma_{k}$.

There exists a $\delta>0$ and an integer $n_{0}$ such that for $0 \leqq|z|<\delta$ and $n>n_{0}, f^{\prime}(z)$ and $R_{n}^{\prime}(z)$ differ from zero and $\log R_{n}^{\prime}(z) \rightarrow \log f^{\prime}(z)$, where we take the determination of the logarithm which is zero for $z=0$. Now

$$
\begin{aligned}
\log R_{n}^{\prime}(z) & =z \sum_{k=1}^{n}\left(2 / \gamma_{k, n}-1 / \alpha_{k, n}-1 / \beta_{k, n}\right) \\
& +\left(z^{2} / 2\right) \sum_{k=1}^{n}\left(2 /\left(\gamma_{k, n}\right)^{2}-1 /\left(\alpha_{k, n}\right)^{2}-1 /\left(\beta_{k, n}\right)^{2}\right)+\cdots
\end{aligned}
$$

Hence $\lim _{n \rightarrow \infty}\left|\sum_{k-1}^{n}\left(2 / \gamma_{k, n}-1 / \alpha_{k, n}-1 / \beta_{k, n}\right)\right|$ exists and is finite. 
Since $\beta_{k, n}<\gamma_{k, n}<0$, we have $-\infty<\lim _{n \rightarrow \infty} \sum_{k=1}^{n}\left(-2 /\left|\gamma_{k, n}\right|-1 / \alpha_{k, n}\right.$ $\left.+1 /\left|\beta_{k, n}\right|\right)<-\lim \sup _{n \rightarrow \infty} \sum_{k=1}^{n}\left(1 /\left|\beta_{k, n}\right|+1 / \alpha_{k, n}\right)<0$. So

$$
\underset{n \rightarrow \infty}{\limsup } \sum_{k=1}^{n} 1 / \alpha_{k, n}<\infty, \quad \underset{n \rightarrow \infty}{\limsup } \sum_{k=1}^{n} 1 /\left|\beta_{k, n}\right|<\infty,
$$

$$
\limsup _{n \rightarrow \infty} \sum_{k=1}^{n} 1 /\left|\gamma_{k, n}\right|<\infty \text {, }
$$

and hence

$$
\sum_{k=1}^{\infty} 1 / \alpha_{k}, \quad \sum_{k=1}^{\infty} 1 /\left|\beta_{k}\right|, \quad \sum_{k=1}^{\infty} 1 /\left|\gamma_{k}\right| \quad \text { all converge. }
$$

Because of (3) the product $\pi(z)=\prod_{k=1}^{\infty}\left(1-z / \alpha_{k}\right)\left(1-z / \beta_{k}\right) /\left(1-z / \gamma_{k}\right)^{2}$ converges, and for $|z|<\delta, \lim _{n \rightarrow \infty} \log R_{n}^{\prime}(z) / \pi(z)=\log f^{\prime}(z) / \pi(z)$.

$$
\begin{aligned}
\log R_{n}^{\prime}(z) / \pi(z)= & \sum_{m=1}^{\infty}\left(z^{m} / m\right)\left\{\left(\sum_{k=1}^{n} 2 /\left(\gamma_{k, n}\right)^{m}-\sum_{k=1}^{\infty} 2 /\left(\gamma_{k}\right)^{m}\right)\right. \\
& -\left(\sum_{k=1}^{n} 1 /\left(\alpha_{k, n}\right)^{m}-\sum_{k=1}^{\infty} 1 /\left(\alpha_{k}\right)^{m}\right) \\
& \left.-\left(\sum_{k=1}^{n} 1 /\left(\beta_{k, n}\right)^{m}-\sum_{k=1}^{\infty} 1 /\left(\beta_{k}\right)^{m}\right)\right\} .
\end{aligned}
$$

Since $\lim _{n \rightarrow \infty} \gamma_{k, n}=\gamma_{k},\left|\lim \sup _{n \rightarrow \infty}\left(\sum_{k=1}^{n} 1 /\left(\gamma_{k, n}\right)^{m}-\sum_{k=1}^{\infty} 1 /\left(\gamma_{k}\right)^{m}\right)\right|$ $=\left|\lim \sup _{n \rightarrow \infty}\left(\sum_{k=n_{0}}^{n} 1 /\left(\gamma_{k, n}\right)^{m}-\sum_{k=n_{0}}^{\infty} 1 /\left(\gamma_{k}\right)^{m}\right)\right|$, and using (2) and (3), we have $\left|\lim \sup _{n \rightarrow \infty}\left(\sum_{k=n_{0}}^{\infty} 1 /\left(\gamma_{k, n}\right)^{m}-\sum_{k=n_{0}}^{\infty} 1 /\left(\gamma_{k}\right)^{m}\right)\right|$ $\leqq 2 \sum_{k=n_{0}}^{n} 1 / k^{m}$ for all $n_{0}$ sufficiently large. So

$$
\lim _{n \rightarrow \infty}\left(\sum_{k=1}^{n} 1 /\left(\gamma_{k, n}\right)^{m}-\sum_{k=1}^{\infty} 1 /\left(\gamma_{k}\right)^{m}\right)=0, \quad m \geqq 2 .
$$

Similarly we have

$$
\lim _{n \rightarrow \infty}\left(\sum_{k=1}^{n} 1 /\left(\alpha_{k, n}\right)^{m}-\sum_{k=1}^{\infty} 1 /\left(\alpha_{k}\right)^{m}\right)=0
$$

and

$$
\lim _{n \rightarrow \infty}\left(\sum_{k=1}^{n} 1 /\left(\beta_{k, n}\right)^{m}-\sum_{k=1}^{\infty} 1 /\left(\beta_{k}\right)^{m}\right)=0 \quad \text { for } m \geqq 2 .
$$

Therefore $\log f^{\prime}(z) / \pi(z)=\delta z, \delta$ real and $f^{\prime}(z)=e^{\delta z} \pi(z)$.

$\pi(z)$ is a canonical product of genus zero, and for

$$
0<\xi_{1} \leqq \arg z \leqq \eta_{1}<\pi, \quad \pi<\xi_{2} \leqq \arg z \leqq \eta_{2}<2 \pi,
$$


it can be proved $[9$, chap. VIII] that $|\pi(z)|=O[\exp (\epsilon|z|)]$ and $1 /|\pi(z)|=O[\exp (\epsilon|z|)]$ for any $\epsilon>0$. Hence

$$
\exp (\delta \Re(z)-\epsilon|z|) \leqq\left|f^{\prime}(z)\right| \leqq \exp (\delta \Re(z)+\epsilon|z|) .
$$

Suppose $\delta>0$ and let $z \rightarrow \infty$ with $\pi / 6 \leqq \arg z \leqq \pi / 3$; we can find $\epsilon>0$ such that $\exp (\delta \Re(z)-\epsilon|z|) \rightarrow \infty$. If $z \rightarrow \infty$ with $2 \pi / 3 \leqq \arg z \leqq 5 \pi / 6$, we can find $\epsilon>0$ such that $\exp (\delta \Re(z)+\epsilon|z|) \rightarrow 0$. Now $f(z)=\int_{0}^{z} f^{\prime}(t) d t$ so that there will exist a curve $\Gamma_{1}$ in $2 \pi / 3 \leqq \arg z \leqq 5 \pi / 6$ extending to $\infty$ along which $|f(z)| \rightarrow$ a finite value $c$, and there will exist a curve $\Gamma_{2}$ in $\pi / 6 \leqq \arg z \leqq \pi / 3$ extending to $\infty$ along which $|f(z)| \rightarrow \infty$. Consider the upper part of the path of reality $C_{2 j}$ and let $z$ move along this path starting at $\alpha_{2 j}$. If $\delta>0$, as $z$ moves along $C_{2 j}$ for $j$ sufficiently large $|f(z)|$ will attain an arbitrarily large value and then decrease to a finite value $c_{1}$ (arbitrarily near $c$ ). But the image $w=f(z)$ will move on the real axis starting at $a_{2 j}$ and moving monotonically to the left to $b_{2 j}$, which is a contradiction and hence $\delta$ cannot be positive. If $\delta<0$ we are not led to a contradiction, for in this case $|f(z)| \rightarrow c$ as $z$ moves along the curve $\Gamma_{2}$ to $\infty$ and $|f(z)| \rightarrow \infty$ as $z$ moves to $\infty$ along $\Gamma_{1}$. So that as $z$ moves counterclockwise on $C_{2 j}$ or $C_{2 j+1},|f(z)|$ will increase from $c_{1}$ to an arbitrarily large value, and the image $w=f(z)$ will move either to the left on the real axis from $a_{2 k}$ or to the right on the real axis from $a_{2 k+1}$. We have proved the following

TheOREM I. Any Riemann surface belonging to the class described in $\$ 2$ is parabolic and the mapping function is given by

$$
f(z)=\int_{0}^{z} f^{\prime}(t) d t, \quad f^{\prime}(z)=e^{\delta z} \prod_{k=1}^{\infty}\left(1-z / \alpha_{k}\right)\left(1-z / \beta_{k}\right) /\left(1-z / \gamma_{k}\right)^{2}
$$

where $\delta$ is a nonpositive real number, $\sum_{k=1}^{\infty} 1 / \alpha_{k}<\infty, \sum_{k=1}^{\infty} 1 /\left|\beta_{k}\right|<\infty$, $\sum_{k=1}^{\infty} 1 /\left|\gamma_{k}\right|<\infty, \cdots, \beta_{k}<\gamma_{k}<\beta_{k-1}<\cdots<\beta_{1}<\gamma_{1}<0<\alpha_{1}<\alpha_{2}$ $<\cdots<\alpha_{k}<\alpha_{k+1}<\cdots$, and the residues of $f^{\prime}(z)$ at the poles $\gamma_{k}$ are all zero.

6. Proof that the members of the class of doubly-connected surfaces are images of the entire plane less two points. Let $\mathcal{F}$, a member of the class of doubly-connected surfaces, be mapped onto the annulus $0 \leqq r<|z|<R \leqq \infty$ by the function $z=\phi(w), w=f(z)$. This mapping will be unique if we require $f(1)=0 \in S_{1}$ and $f^{\prime}(1)>0$. By the methods mentioned in $\$ 4$ the following can be obtained.

(1) $f(z)$ is real for $z$ real.

(2) The image of the branch point over $b_{j}$ is $\beta_{j}$, the image of the branch point over $a_{j}$ is $\alpha_{j}$, the image of the point of $\mathcal{F}$ on $S_{j}$ over 
$w=\infty$ is $z=\gamma_{j}, j= \pm 1, \pm 2, \pm 3, \cdots$, with $\cdots \beta_{k}<\gamma_{k}<\beta_{k-1}<\cdots$ $<\beta_{1}<\gamma_{1}<\beta_{-1}<\gamma_{-1}<\cdots<\beta_{-k}<\gamma_{-k}<\beta_{-k-1}<\cdots<-r<0<r$ $<\cdots<\alpha_{-k}<\alpha_{-k+1}<\cdots<\alpha_{-1}<1<\alpha_{k}<\alpha_{k+1}, k=1,2,3, \cdots$.

(3) $S_{1}$ is mapped on a portion of the annulus bounded by two nonintersecting, simple, closed curves $C_{1}$ and $C_{-1}$, symmetric about the real axis with $C_{1}$ cutting the real axis in $\alpha_{1}$ and $\beta_{1}$ only, and $C_{-1}$ cutting the real axis in $\alpha_{-1}$ and $\beta_{-1}$ only. $S_{j}, j \neq 1$, is mapped on a portion of $0 \leqq r<|z|<R \leqq \infty$ bounded by two nonintersecting, simple, closed curves $C_{j}$ and $C_{j-1}$, symmetric about the real axis with $C_{j}$ cutting the real axis in $\alpha_{j}$ and $\beta_{j}$ only, and $C_{j-1}$ cutting the real axis in $\alpha_{j-1}$ and $\beta_{j-1}$ only.

That $r=0$ and $R=\infty$ follows from the parabolicity of the surfaces of the simply-connected class. Consider $\mathcal{F}$ as made up of two surfaces $\mathcal{F}_{1}$ and $\mathcal{F}_{2}: \mathcal{F}_{1}$ consisting of sheets $S_{1}, S_{2}, S_{3}, \cdots, \mathcal{F}_{2}$ consisting of sheets $S_{-1}, S_{-2}, S_{-3}, \cdots$, with $\mathcal{F}_{1}$ and $\mathcal{F}_{2}$ joined along the cut from $a_{-1}$ to $b_{-1}$. Then $\mathcal{F}_{1}$ is mapped on a domain $\Delta_{1}$ of the $z$-plane bounded by the curve $C_{-1}$ and $|z|=R$. But by the results of $\$ 4, \mathcal{F}_{1}$ can be mapped onto the plane $|\zeta|<\infty$ with the cut over $\left(a_{-1}, b_{-1}\right)$ being mapped into a portion of the real axis $(a, b)$. Hence the punched $\zeta$-plane cut along the real axis from $a$ to $b$ can be mapped onto $\Delta_{1}$ by a regular function with the cut $(a, b)$ corresponding to $C_{-1}$. But then $R$ must be infinite, for otherwise the circle $|z|=R<\infty$ would be the image of the point $\zeta=\infty$ by a regular function, which is impossible. By a similar argument we can show $r=0$. Therefore $\mathcal{f}$ can be mapped on the $z$-plane less the two points $z=0, z=\infty$.

7. Structure of the mapping function for surfaces of the second class. We have the portion of the z-plane exterior to the curve $C_{-1}$ mapped one-to-one on $\mathcal{F}_{1}$ by $w=f(z)$. But from the results of $\$ 4$ we have the $\zeta$-plane less the point $\zeta=\infty$ and the segment $C^{\prime}$ of the real axis from $\phi\left(a_{-1}\right)$ to $\phi\left(b_{-1}\right)$ mapped one-to-one on $\mathcal{f}_{1}$ by $w=G_{1}(\zeta)$. Hence, the punched $\zeta$-plane cut along $C^{\prime}$ can be mapped schlichtly by $z=h(\zeta)$ onto the portion of the $z$-plane exterior to $C_{-1}$ in such a way that $\zeta=0$ corresponds to $z=1, C^{\prime}$ corresponds to $C_{-1}$, and $\zeta=\infty$ corresponds to $z=\infty$, i.e., $h(\zeta)$ must have a simple pole at $\zeta=\infty$. So $h(\zeta)=\zeta \psi(\zeta)$ where $\psi(\zeta)$ is regular at $\zeta=\infty$ and $\psi(\infty) \neq 0$, or $h(\zeta)$ $=c \zeta+q(\zeta)$ where $q(\zeta)$ is regular at $\zeta=\infty$ and hence bounded in the neighborhood of $\zeta=\infty$. So we can write $h(\zeta)=\zeta+O(1)(\zeta \rightarrow \infty)$. So $G_{1}(\zeta)=f[h(\zeta)], G_{1}^{\prime}(\zeta)=f^{\prime}(z) h^{\prime}(\zeta), h^{\prime}(\zeta) \neq 0$ for $\zeta$ sufficiently large. From the results of $\$ 5$ we know that if the zeros of $G_{1}^{\prime}(\zeta)$ are denoted by $\zeta_{k}$, then $\sum_{k=1}^{\infty} 1 /\left|\zeta_{k}\right|<\infty$. Since $z \sim c \zeta$ as $\zeta \rightarrow \infty$, the zeros $\alpha_{k}$ and $\beta_{k}$ of $f^{\prime}(z)$ are such that 


$$
\sum_{k=1}^{\infty} 1 /\left|\alpha_{k}\right|<\infty, \quad \sum_{k=1}^{\infty} 1 /\left|\beta_{k}\right|<\infty .
$$

In a similar manner we have the $\zeta$-plane cut along a segment $C^{\prime \prime}$ of the real axis mapped one-to-one by $z=g(\zeta)$ onto the portion of the z-plane interior to $C_{1}$ in such a way that as $\zeta \rightarrow \infty, g(\zeta) \rightarrow 0$, i.e., $g(\zeta)$ must have a simple zero at $\zeta=\infty$. So $g(\zeta)=\psi(\zeta) / \zeta$ where $\psi(\infty) \neq 0$ and $\psi(\zeta)$ is regular at $\zeta=\infty$, or $g(\zeta)=c / \zeta+p(\zeta)$ where $p(\zeta)$ is $O\left(1 /|\zeta|^{2}\right)$ as $\zeta \rightarrow \infty$. Then if $w=f(z)$ is the function mapping the interior of $C_{1}$ onto the surface $\mathcal{F}_{2}$ and $w=G_{2}(\zeta)$ is the function mapping the $\zeta$-plane less the point $\zeta=\infty$ and the cut $C^{\prime \prime}$ onto $\mathcal{F}_{2}, G_{2}(\zeta)$ $=f[g(\zeta)], G_{2}^{\prime}(\zeta)=f^{\prime}(z) g^{\prime}(\zeta), g^{\prime}(\zeta) \neq 0$ for $\zeta$ sufficiently large. Again from the results of $\$ 5$ we know that if the zeros of $G_{2}^{\prime}(\zeta)$ are $\zeta_{k}$, then $\sum_{k=1}^{\infty} 1 /\left|\zeta_{k}\right|<\infty$ so that, since $z \sim c / \zeta$ as $\zeta \rightarrow \infty$, the zeros of $f^{\prime}(z)$, $\alpha_{-k}, \beta_{-k}$, are such that $\sum_{k=1}^{\infty}\left|\alpha_{-k}\right|<\infty, \sum_{k-1}^{\infty}\left|\beta_{-k}\right|<\infty$.

Consider the surface $\mathcal{F}$ as the limit of a sequence of surfaces $\left\{\mathcal{F}_{n}\right\}$, where $\mathcal{F}_{n}$ consists of the $2 n+1$ sheets $S_{-n}, S_{-n+1}, \cdots, S_{-1}, S_{1}, \cdots$, $S_{n+1}$ with the cuts $\left(a_{-n-1}, b_{-n-1}\right)$ and $\left(a_{n+1}, b_{n+1}\right)$ healed. $\mathcal{F}_{n}$ is the Riemann surface of the inverse of a rational function $w=R_{n}(z)$, $R_{n}(z)=P_{n}(z) / Q_{n}(z), \quad R_{n}(1)=0 \in S_{1}, \quad R_{n}^{\prime}(1)>0, \quad R_{n}(\infty)=\infty \in S_{n+1}$, $R_{n}(0)=\infty \in S_{-n}$, where $P_{n}(z)$ is a polynomial of degree $2 n+1$ and $Q_{n}(z)$ is a polynomial of degree $2 n$.

$$
R_{n}^{\prime}(z)=K_{n} \frac{\prod_{k=1}^{n}\left(1-z / \alpha_{k, n}\right)\left(1-z / \beta_{k, n}\right)\left(1-\alpha_{-k, n} / z\right)\left(1-\beta_{-k, n} / z\right)}{\prod_{k=1}^{n}\left(1-z / \gamma_{k, n}\right)^{2} \prod_{k=1}^{n-1}\left(1-\gamma_{-k, n} / z\right)^{2}}
$$

$K_{n}>0$, where $\alpha_{j, n}, \beta_{j, n}$ are the images of the points in the surface over $a_{j}, b_{j}$, and $\gamma_{j, n}$ is the image of the point over $\infty$ in sheet $S_{j}$, and the residues of $R_{n}^{\prime}(z)$ at the poles are zero. $R_{n}(z)=\int_{1}^{z} R_{n}^{\prime}(t) d t$. Now $\zeta=\phi\left[R_{n}(z)\right]$ maps the $z$-plane cut from $z=\alpha_{n, n}$ to $\infty$ and from $z=\alpha_{-n, n}$ to zero along the real axis schlichtly onto a domain bounded by $C_{n+1}, C_{-n-1}$ cut along the segments

$$
\left(\alpha_{n}, \alpha_{n+1}\right), \quad\left(\alpha_{-n-1}, \alpha_{-n}\right), \quad\left(\beta_{n+1}, \gamma_{n+1}\right), \quad \text { and }\left(\gamma_{-n}, \beta_{-n-1}\right) .
$$

So using the fact that $r=0$ and $R=\infty$, and applying the theorem of Carathéodory mentioned in $\$ 5$, we have

$$
\lim _{n \rightarrow \infty} R_{n}(z)=f(z) \text { and } \lim _{n \rightarrow \infty} R_{n}^{\prime}(z)=f^{\prime}(z) \text { uniformly. }
$$

Hence $\lim _{n \rightarrow \infty} \alpha_{j, n}=\alpha_{j}, \lim _{n \rightarrow \infty} \beta_{j, n}=\beta_{j}, \lim _{n \rightarrow \infty} \gamma_{j, n}=\gamma_{j}, j= \pm 1, \pm 2$, $\pm 3, \cdots$, and we know 


$$
\begin{array}{lll}
\sum_{k=1}^{\infty} \frac{1}{\left|\alpha_{k}\right|} ; & \sum_{k=1}^{\infty} \frac{1}{\left|\beta_{k}\right|} ; & \sum_{k=1}^{\infty} \frac{1}{\left|\gamma_{k}\right|} ; \\
\sum_{k=1}^{\infty}\left|\alpha_{-k}\right| ; & \sum_{k=1}^{\infty}\left|\beta_{-k}\right| ; & \sum_{k=1}^{\infty}\left|\gamma_{-k}\right|
\end{array}
$$

all converge. For $|z-1|<\rho$ and $n>n_{0}$ we have $f^{\prime}(z) \neq 0$ and $R_{n}^{\prime}(z) \neq 0$, so for these values of $z, \lim _{n \rightarrow \infty} \log R_{n}^{\prime}(z)=\log f^{\prime}(z)$, taking the determination of the logarithm which is real for $z=1$. If we choose $k_{0}$ so that, for $|z-1|<\rho$ and $n>n_{0}, \max \left[\left|z / \beta_{k_{0}}\right| ;\left|\beta_{-k_{0}, n} / z\right|\right]<1$ we can write

$$
\begin{aligned}
\log R_{n}^{\prime}(z)= & \log \prod_{k=1}^{k_{0}} \frac{\left(1-z / \alpha_{k, n}\right)\left(1-z / \beta_{k, n}\right)\left(1-\alpha_{-k, n} / z\right)\left(1-\beta_{-k, n} / z\right)}{\left(1-z / \gamma_{k, n}\right)^{2}\left(1-\gamma_{-k, n} / z\right)^{2}} \\
& +z \sum_{k=k_{0}}^{n}\left(2 / \gamma_{k, n}-1 / \alpha_{k, n}-1 / \beta_{k, n}\right) \\
& +\frac{z^{2}}{2} \sum_{k=k_{0}}^{n}\left[2 /\left(\gamma_{k, n}\right)^{2}-1 /\left(\alpha_{k, n}\right)^{2}-1 /\left(\beta_{k, n}\right)^{2}\right]+\cdots \\
& +\frac{1}{z} \sum_{k=k_{0}}^{n-1}\left(2 \gamma-k, n-\alpha_{-k, n}-\beta_{-k, n}\right) \\
& +\frac{1}{2 z^{2}} \sum_{k=k_{0}}^{n-1}\left[2\left(\gamma_{-k, n}\right)^{2}-\left(\alpha_{-k, n}\right)^{2}-\left(\beta_{-k, n}\right)^{2}\right]+\cdots \\
& -\left[\frac{\alpha-n, n}{z}+\frac{\left(\alpha_{-n, n}\right)^{2}}{2 z^{2}}+\cdots\right] \\
& -\left[\frac{\beta_{-n, n}}{z}+\frac{\left(\beta_{-n, n}\right)^{2}}{2 z^{2}}+\cdots\right] \\
& +\log K_{n .}
\end{aligned}
$$

Hence $\lim _{n \rightarrow \infty}\left|\sum_{k=z_{0}}^{k}\left(2 / \gamma_{k, n}-1 / \alpha_{k, n}-1 / \beta_{k, n}\right)\right|$ and

$$
\lim _{n \rightarrow \infty}\left|\sum_{k=k_{0}+1}^{n}\left(2 \gamma_{-k+1, n}-\alpha_{-k, n}-\beta_{-k, n}\right)-\alpha_{k_{0}, n}-\beta_{k_{0}, n}\right|
$$

exist and are finite. We have $0>\gamma_{k, n}>\beta_{k, n}$ or $-1 /\left|\gamma_{k, n}\right|<-1 /\left|\beta_{k, n}\right|$. Thus we have $-\infty<\lim _{n \rightarrow \infty} \sum_{k=k_{0}}^{n}\left(-2 /\left|\gamma_{k, n}\right|-1 / \alpha_{k, n}+1 /\left|\beta_{k, n}\right|\right)$ $<\lim \sup _{n \rightarrow \infty}\left[-\sum_{k=k_{0}}^{n}\left(1 / \alpha_{k, n}+1 /\left|\beta_{k, n}\right|\right)\right]<0$. Therefore

$$
\underset{n \rightarrow \infty}{\limsup } \sum_{k=k_{0}}^{n} 1 /\left|\beta_{k, n}\right|<\infty, \quad \limsup _{n \rightarrow \infty} \sum_{k=k_{0}}^{n} 1 / \alpha_{k, n}<\infty,
$$




$$
\underset{n \rightarrow \infty}{\lim \sup _{i}} \sum_{k=k_{0}}^{n} 1 /\left|\gamma_{k, n}\right|<\infty \text {. }
$$

Also $0>\beta_{-k, n}>\gamma_{-k+1, n}$ or $-\left|\gamma_{-k+1, n}\right|<-\left|\beta_{-k, n}\right|$, hence

$$
\begin{aligned}
-\infty< & \lim _{n \rightarrow \infty}\left[\sum_{k=k_{0}+1}^{n}\left(-2\left|\gamma_{-k+1, n}\right|-\alpha_{-k, n}+\left|\beta_{-k, n}\right|\right)\right. \\
& \left.-\alpha_{-k_{0}, n}+\left|\beta_{-k_{0}, n}\right|\right] \\
& <\lim _{n \rightarrow \infty} \sup _{n}-\left[\sum_{k=k_{0}+1}^{n}\left(\alpha_{-k, n}+\left|\beta_{-k, n}\right|\right)+\alpha_{-k_{0}, n}-\left|\beta_{-k_{0}, n}\right|\right] .
\end{aligned}
$$

Therefore $\lim \sup _{n \rightarrow \infty} \sum_{k-k_{0}}^{n} \alpha_{-k, n}<\infty, \lim \sup _{n \rightarrow \infty} \sum_{k=k_{0}}^{n}\left|\beta_{-k, n}\right|<\infty$, $\lim \sup _{n \rightarrow \infty} \sum_{k=k_{0}}^{n-1}\left|\gamma_{-k, n}\right|<\infty$. Let

$$
\pi(z)=\frac{\prod_{k=1}^{\infty}\left(1-z / \alpha_{k}\right)\left(1-z / \beta_{k}\right)\left(1-\alpha_{-k} / z\right)\left(1-\beta_{-k} / z\right)}{\prod_{k=1}^{\infty}\left(1-z / \gamma_{k}\right)^{2}\left(1-\gamma_{-k} / z\right)^{2}} .
$$

Then

$$
\begin{aligned}
\log \left[R_{n}^{\prime}(z) / \pi(z)\right] & =\sum_{m=1}^{\infty}\left\{\frac { z ^ { m } } { m } \left[\left(\sum_{k=k_{0}}^{n} \frac{2}{\left(\gamma_{k, n}\right)^{m}}-\sum_{k=k_{0}}^{\infty} \frac{2}{\left(\gamma_{k}\right)^{m}}\right)\right.\right. \\
& -\left(\sum_{k=k_{0}}^{n} \frac{1}{\left(\alpha_{k, n}\right)^{m}}-\sum_{k=k_{0}}^{\infty} \frac{1}{\left(\alpha_{k}\right)^{m}}\right) \\
& \left.\left.-\left(\sum_{k=k_{0}}^{n} \frac{1}{\left(\beta_{k, n}\right)^{m}}-\sum_{k=k_{0}}^{\infty} \frac{1}{\left(\beta_{k}\right)^{m}}\right)\right]\right\} \\
& +\sum_{m=1}^{\infty}\left\{\frac { 1 } { m z ^ { m } } \left[\left(\sum_{k=k_{0}}^{n-1} 2\left(\gamma_{-k, n}\right)^{m}-\sum_{k=k_{0}}^{\infty} 2\left(\gamma_{-k}\right)^{m}\right)\right.\right. \\
& -\left(\sum_{k=k_{0}}^{n-1}\left(\alpha_{-k, n}\right)^{m}-\sum_{k=k_{0}}^{\infty}\left(\alpha_{-k}\right)^{m}\right) \\
& \left.\left.-\left(\sum_{k=k_{0}}^{n-1}\left(\beta_{-k, n}\right)^{m}-\sum_{k=k_{0}}^{\infty}\left(\beta_{-k}\right)^{m}\right)\right]\right\} \\
& +\log K_{n}+T_{n}(z),
\end{aligned}
$$

where $T_{n}(z)=o(1)$ as $n \rightarrow \infty$.

As in $\$ 5$ it can be shown that, for $m \geqq 2$, 


$$
\begin{aligned}
& \lim _{n \rightarrow \infty}\left(\sum_{k=k_{0}}^{n} 1 /\left(\gamma_{k, n}\right)^{m}-\sum_{k=k_{0}}^{\infty} 1 /\left(\gamma_{k}\right)^{m}\right), \lim _{n \rightarrow \infty}\left(\sum_{k=k_{0}}^{n-1}(\gamma-k, n)^{m}-\sum_{k=k_{0}}^{\infty}(\gamma-k)^{m}\right), \\
& \lim _{n \rightarrow \infty}\left(\sum_{k=k_{0}}^{n} 1 /\left(\alpha_{k, n}\right)^{m}-\sum_{k=k_{0}}^{\infty} 1 /\left(\alpha_{k}\right)^{m}\right), \lim _{n \rightarrow \infty}\left(\sum_{k=k_{0}}^{n}\left(\alpha_{-k, n}\right)^{m}-\sum_{k=k_{0}}^{\infty}\left(\alpha_{-k}\right)^{m}\right), \\
& \lim _{n \rightarrow \infty}\left(\sum_{k=k_{0}}^{n} 1 /\left(\beta_{k, n}\right)^{m}-\sum_{k=k_{0}}^{\infty} 1 /\left(\beta_{k}\right)^{m}\right), \lim _{n \rightarrow \infty}\left(\sum_{k=k_{0}}^{n}\left(\beta_{-k, n}\right)^{m}-\sum_{k=k_{0}}^{\infty}\left(\beta_{-k}\right)^{m}\right)
\end{aligned}
$$

all exist and are zero, and we have

$$
\begin{aligned}
\log f^{\prime}(z) / \pi(z) & \\
= & \lim _{n \rightarrow \infty} \log R_{n}^{\prime}(z) / \pi(z) \\
= & z \lim _{n \rightarrow \infty}\left\{\left(\sum_{k=k_{0}}^{n} 2 / \gamma_{k, n}-\sum_{k=k_{0}}^{\infty} 2 / \gamma_{k}\right)-\left(\sum_{k=k_{0}}^{n} 1 / \alpha_{k, n}-\sum_{k=k}^{\infty} 1 / \alpha_{k}\right)\right. \\
& \left.-\left(\sum_{k=k_{0}}^{n} 1 / \beta_{k, n}-\sum_{k=k_{0}}^{\infty} 1 / \beta_{k}\right)\right\} \\
& +(1 / z) \lim _{n \rightarrow \infty}\left\{\left(\sum_{k=k_{0}}^{n-1} 2 \gamma_{-k, n}-\sum_{k=k_{0}}^{\infty} 2 \gamma_{-k}\right)\right. \\
& \left.-\left(\sum_{k=k_{0}}^{n} \alpha_{-k, n}-\sum_{k=k_{0}}^{\infty} \alpha_{-k}\right)-\left(\sum_{k=k_{0}}^{n} \beta_{-k, n}-\sum_{k=k_{0}}^{\infty} \beta_{-k}\right)\right\} \\
& +\log K,
\end{aligned}
$$

$\log f^{\prime}(z) / \pi(z)=\sigma_{1} z+\sigma_{2} / z+\log K$ where $K$ and $\sigma_{1}, \sigma_{2}$ are real. So $f^{\prime}(z)=K\left[\exp \left(\sigma_{1} z+\sigma_{2} / z\right)\right] \pi(z)$.

With an argument similar to the one used in $\$ 5$ it may be shown that $\sigma_{1}$ and $\sigma_{2}$ cannot be positive, so we have

Theorem II. Any Riemann surface belonging to the class described in $\S 3$ is the image of the $z$-plane less the two points $z=\infty$ and $z=0$ by $a$ function $w=f(z)$ where

$$
\begin{aligned}
f(z) & =\int_{1}^{z} f^{\prime}(t) d t \\
f^{\prime}(z) & =K \exp \left(\sigma_{1} z+\sigma_{2} / z\right) \prod_{k=1}^{\infty} \frac{\left(1-z / \alpha_{k}\right)\left(1-z / \beta_{k}\right)\left(1-\alpha_{-k} / z\right)\left(1-\beta_{-k} / z\right)}{\left(1-z / \gamma_{k}\right)^{2}\left(1-\gamma_{-k} / z\right)^{2}}
\end{aligned}
$$

where $K>0, \sigma_{1}, \sigma_{2}, \alpha_{j}, \beta_{j}, \gamma_{j}(j=1, \pm 1, \pm 2, \pm 3, \cdots)$ are real with $\cdots \beta_{k}<\gamma_{k}<\beta_{+k-1}<\cdots<\beta_{1}<\gamma_{1}<\beta_{-1}<\gamma_{-1}<\cdots<\beta_{-k}<\gamma_{-k}<$$$
\cdots<0<\cdots<\alpha_{-k-1}<\alpha_{-k}<\cdots<\alpha_{-1}<\alpha_{1} \cdots<\alpha_{k} \cdots \text {, }
$$ 


$$
\begin{array}{lll}
\sum_{k=1}^{\infty} 1 / \alpha_{k}<\infty ; & \sum_{k=1}^{\infty} 1 /\left|\beta_{k}\right|<\infty ; & \sum_{k=1}^{\infty} 1 /\left|\gamma_{k}\right|<\infty ; \\
\sum_{k=1}^{n} \alpha_{-k}<\infty ; & \sum_{k=1}^{\infty}\left|\beta_{-k}\right|<\infty ; & \sum_{k=1}^{\infty}\left|\gamma_{-k}\right|<\infty,
\end{array}
$$

and the residues of $f^{\prime}(z)$ at the poles are all zero, and $\sigma_{1}, \sigma_{2}$ are nonpositive.

\section{Partial converse of Theorem I.}

TheOREM III. Let $w=f(z)$ be meromorphic in $|z|<\infty$ with $f(0)=0$, $f^{\prime}(0)=1$. If $f(z)$ has the form $f(z)=z e^{\delta z} \prod_{k=1}^{\infty}\left(1-z / c_{k}\right) /\left(1-z / \gamma_{k}\right)$ where $c_{k}, \gamma_{k}, \delta$ are real, $\delta \leqq 0, \gamma_{k+1}<\gamma_{k}<0<c_{k}<c_{k+1}(k=1,2, \cdots)$ with $\sum_{k=1}^{\infty} 1 / c_{k}<\infty, \sum_{k=1}^{\infty} 1 /\left|\gamma_{k}\right|<\infty$, then the Riemann surface of the inverse of $f(z)$ is a surface of the class described in $\$ 2$.

Note that if in $\$ 2$ the sequence $\left\{a_{k}\right\}$ is required to satisfy $a_{2 k}<0$ $<a_{2 k \pm 1}$, the function of Theorem I would satisfy the hypotheses of the above. The proof of Theorem III rests on the construction of a sequence of rational functions $\left\{R_{n}(z)\right\}$ which converges uniformly to $f(z)$ and whose members have derivatives of the same form as the $R_{n}^{\prime}(z)$ in $\$ 2$. The author has been unable to show that such a construction is possible without restricting the location of the zeros of $f(z)$ and so has been unable to give a proof of the complete converse of Theorem I although he conjectures its validity.

We distinguish two cases.

Case I: $\delta=0$. We may construct a sequence of rational functions $\left\{R_{n}(z)\right\}$ which converges uniformly to $f(z)$ by setting $R_{n}(z)$ $=z \prod_{k-1}^{n}\left(1-z / c_{k}\right) /\left(1-z / \gamma_{k}\right) . R_{n}(z)$ has simple poles at $z=\gamma_{k}$, $k=1,2,3, \cdots, n$, and at $z=\infty$, and the residues at adjacent poles will differ in sign. Therefore there are at least $n$ points $z=\beta_{k, n}$, $k=1,2,3, \cdots, n$, on the negative real axis such that $R_{n}^{\prime}\left(\beta_{k, n}\right)=0$ and the points will be distributed as follows: $-\infty<\beta_{n, n}<\gamma_{n}<\beta_{n-1, n}$ $<\cdots<\gamma_{k+1}<\beta_{k, n}<\gamma_{k}<\cdots<\beta_{1, n}$. There will also be at least $n$ points $z=\alpha_{k, n}, k=1,2,3, \cdots, n$, on the positive real axis such that $R_{n}^{\prime}\left(\alpha_{k, n}\right)=0$ and these points will be distributed as follows: $0<\alpha_{1, n}$ $<c_{1}<\alpha_{2, n}<\cdots<c_{k-1}<\alpha_{k, n}<c_{k}<\cdots<\alpha_{n, n}<c_{n}$. Hence

$$
R_{n}^{\prime}(z)=\prod_{k=1}^{n}\left(1-z / \alpha_{k, n}\right)\left(1-z / \beta_{k, n}\right) /\left(1-z / \gamma_{k}\right)^{2}
$$

and $R_{n}^{\prime}(z) \rightarrow f^{\prime}(z)$ uniformly.

It is obvious that the paths of reality consist of $n$ simple, closed curves, symmetric about the real axis and intersecting the real axis 
at the points $z=\alpha_{k, n}, z=\beta_{k, n}, k=1,2,3, \cdots, n$. We assert that $\beta_{j, n}, \beta_{k, n}, k \neq j$, cannot lie on the same path of reality. For suppose that $\beta_{j, n}, \beta_{k, n}$ are both on the same path of reality $C$, then:

(1) If $k, j$ are both odd or both even there will be an odd number of branch points between $\beta_{j, n}$, and $\beta_{k, n}$ and hence at least one path of reality would have to cross $C$ at some point not on the real axis, which is impossible.
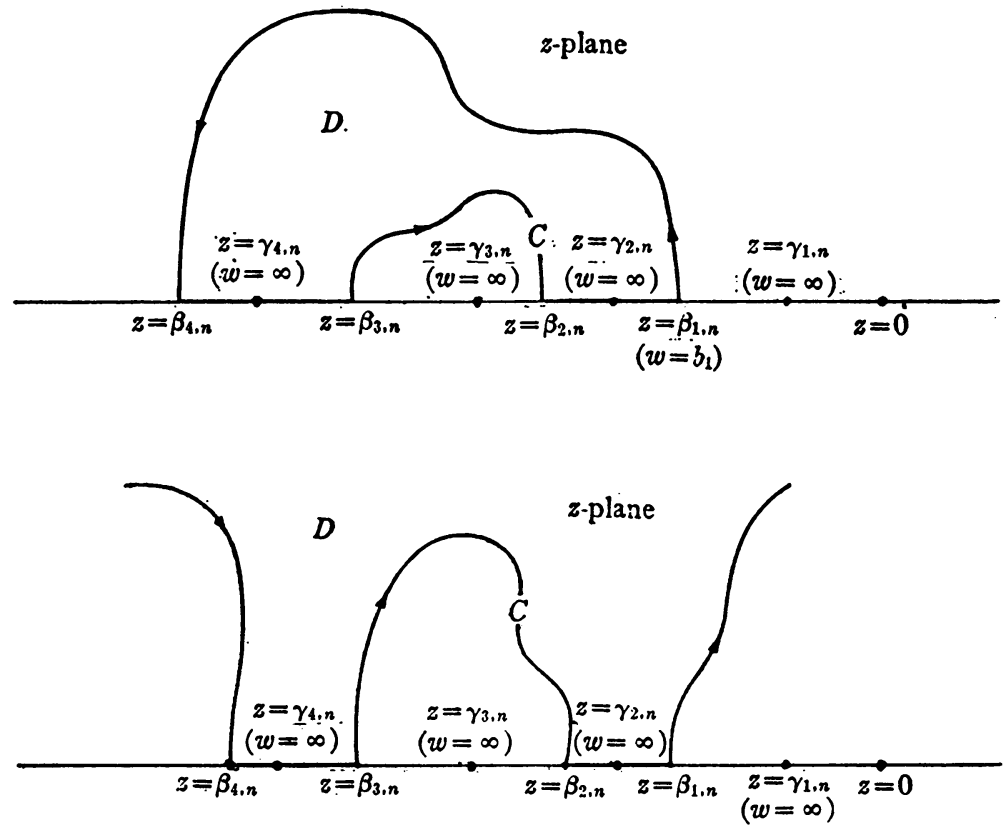

FIG. 1

(2) If $k$ and $j$ are not both odd or both even, as $z$ traverses the path of reality around the domain $D$ interior to the path, $R_{n}(z)$ would take on all real values twice (see Fig. 1), which is impossible. It is now apparent that the path of reality $C_{j}$ through $\beta_{j, n}$ will intersect the real axis in the point $\alpha_{j, n}, j=1,2,3, \cdots, n$.

$R_{n}(z)=P_{n}(z) / Q_{n}(z)$ where $P_{n}(z)$ is a polynomial of degree $n+1$ and $Q_{n}(z)$ is a polynomial of degree $n$. Since $\overline{R_{n}(z)}=R_{n}(\bar{z})$ and $\overline{Q_{n}(z)}=Q_{n}(\bar{z})$ the equation of the paths of reality is given by

$$
2 i \Im\left[R_{n}(z)\right]=P_{n}(z) / Q_{n}(z)-P_{n}(\bar{z}) / Q_{n}(\bar{z})=0
$$

or

$$
F(x, y)=P_{n}(x+i y) Q_{n}(x-i y)-P_{n}(x-i y) Q_{n}(x+i y)=0 .
$$

From considerations of degree we see that any line arg $z=\theta_{0}, \theta_{0} \neq 0, \pi$, 
will intersect the upper part of the paths of reality only once and the lower part of each of the paths of reality only once.

Collecting the foregoing we have: For any $n, R_{n}(z)$ is real for $z$ on the real axis and for $z$ on the noninteresecting, simple, closed curves $C_{1, n}, C_{2, n}, \cdots, C_{n, n}$ symmetric about the real axis with $C_{k, n}$ intersecting the real axis in the points $z=\alpha_{k, n}, z=\beta_{k, n}$. Furthermore, the curves $C_{k, n}$ are star-shaped (any ray from the origin intersects each curve only once).

If we denote by $\alpha_{k}$ and $\beta_{k}$ the zeros of $f^{\prime}(z)$, then $\alpha_{k, n} \rightarrow \alpha_{k}$ and $\beta_{k, n} \rightarrow \beta_{k}$ as $n \rightarrow \infty$, and $C_{k, n}$ will tend to a limiting curve $C_{k}$ which is star-shaped, symmetric about the real axis, and passes through the points $z=\alpha_{k}$ and $z=\beta_{k}$. We wish to show that the curve $C_{k}$ has no points at $z=\infty$. Consider the domain $D_{k, n}$ which contains $C_{k, n}$ and is bounded by $C_{k+1, n}$ and $C_{k-1, n} . D_{k, n}$ is mapped by $R_{n}(z)$ one-to-one onto a two-sheeted surface $\Sigma_{k, n}$ consisting of two slit copies of the $w$ sphere $S^{\prime}$ and $S^{\prime \prime} . S^{\prime}$ is slit between $R_{n}\left(\alpha_{k-1, n}\right)=a_{k-1, n}$ and $R_{n}\left(\beta_{k-1, n}\right)$ $=b_{k-1, n}$ and between $R_{n}\left(\alpha_{k, n}\right)=a_{k, n}$ and $R_{n}\left(\beta_{k, n}\right)=b_{k, n}$. $S^{\prime \prime}$ is slit between $a_{k, n}$ and $b_{k, n}$ and between $R_{n}\left(\alpha_{k+1, n}\right)=a_{k+1, n}$ and $R_{n}\left(\beta_{k+1, n}\right)$ $=b_{k+1, n}$. The sheets are joined along their cuts from $a_{k, n}$ to $b_{k, n}$, and

$$
R_{n}\left(\frac{\alpha_{k}+\alpha_{k+1}}{2}\right)=a \in S^{\prime \prime}, \quad R_{n}^{\prime}\left(\frac{\alpha_{k}+\alpha_{k+1}}{2}\right)=K
$$

$\Sigma_{k, n}$ can in turn be mapped by $\zeta=G(w)$ onto the domain $\Delta_{k, n}$ which is the $\zeta$-plane slit from $G\left(a_{k-1, n}\right)$ to $G\left(b_{k-1, n}\right)$ and from $G\left(a_{k+1, n}\right)$ to $G\left(b_{k+1, n}\right)$. The segment $\left(a_{k, n}, b_{k, n}\right)$ is mapped onto a closed curve $\Gamma_{k, n}$ and $G\left(a \in S^{\prime \prime}\right)=0, G^{\prime}\left(a \in S^{\prime \prime}\right)=1$. The sequence of domains $\Delta_{k, n}$ converges to its kernel $\Delta_{k}$ which is the $\zeta$-plane cut from $G\left(a_{k-1}\right)$ to $G\left(b_{k-1}\right)$ and from $G\left(a_{k+1}\right)$ to $G\left(b_{k+1}\right)$ where $a_{j}=\lim _{n \rightarrow \infty} R_{n}\left(\alpha_{j}\right)$, $b_{j}=\lim _{n \rightarrow \infty} R_{n}\left(\beta_{j}\right)$. Since the sequence of functions $\left\{G\left[R_{n}(z)\right]\right\}$ is schlicht and converges, and since $C_{k, n}$ converges to $C$, we may apply the variation of Carathéodory's theorem mentioned in $\$ 5$ and find that $f(z)$ is regular at every point of $C_{k}$ and hence $C_{k}$ cannot have any ends at $z=\infty$. So the paths of reality of $f(z)$ are the real axis and the star-shaped curves $C_{k}$, symmetric about the real axis, intersecting the real axis in $z=\alpha_{k}$ and $z=\beta_{k}$ with $C_{k}$ having no points at $z=\infty$. Consider the domain $D$ bounded by the upper halves of $C_{j}$ and $C_{j+1}$ and the segments $\left(\alpha_{j}, \alpha_{j+1}\right)$ and $\left(\beta_{j}, \beta_{j+1}\right)$. In this domain $\Im(w)$ has the constant sign $(-1)^{i}$ and hence in $D[w] \neq(-1)^{j+1} i$. The real part of $w$ varies monotonically from $(-1)^{j+1} \infty$ to $(-1)^{j} \infty$ as $z$ traverses the boundary of $D$ in the positive direction starting from $z=\gamma_{j+1}$. Applying the theorem of Darboux we have: $D$ is mapped schlichtly by $w=f(z)$ onto the half-plane $(-1)^{i} \Im(w)>0$. Therefore, if $\delta=0$, the 
surface of the inverse of $w=f(z)$ is a surface of the first class.

Case II: $\delta<0$. We may construct a sequence of rational functions $\left\{R_{n}(z)\right\}$ which converges uniformly to $f(z)$ in any closed bounded region by setting $R_{n}(z)=z\left(1+\delta z / \lambda_{n}\right)^{\lambda_{n}} \prod_{k=1}^{n}\left(1-z / c_{k}\right) /\left(1-z / \gamma_{k}\right)$ where $\left\{\lambda_{n}\right\}$ is a sequence of positive integers increasing to infinity and chosen so that $\left|\lambda_{n} / \delta\right|>c_{n}$. Then $R_{n}^{\prime}(z)=\left(1+\delta z / \lambda_{n}\right)^{\lambda_{n}-1} M_{n}(z) / N_{n}(z)$ where $N_{n}(z)=\prod_{k=1}^{n}\left(1-z / \gamma_{k}\right)^{2}$ and $M_{n}(z)$ is a polynomial of degree $2 n+1$. By the same argument used in the preceding paragraph we find that $R_{n}^{\prime}(z)$ has at least $2 n$ zeros at points $\alpha_{k, n}, \beta_{k, n}, k=1,2,3, \cdots, n$, with $\beta_{n, n}<\gamma_{n}<\cdots<\beta_{k, n}<\gamma_{k}<\cdots<\beta_{1, n}<\gamma_{1}<0<\alpha_{1, n}<c_{1}<\alpha_{2, n}$ $<\cdots<\alpha_{n, n}<c_{n}$. Since $R_{n}(z)$ is zero when $z=-\lambda_{n} / \delta$, there is at least one zero of $R_{n}^{\prime}(z)$ at a point $z=\epsilon_{n}$ where $c_{n}<\epsilon_{n}<-\lambda_{n} / \delta$. Since the degree of $M_{n}(z)$ is $2 n+1$ we can write

$$
R_{n}^{\prime}(z)=\left(1+\delta z / \lambda_{n}\right)^{\lambda_{n}-1}\left(1-z / \epsilon_{n}\right) \prod_{k=1}^{n}\left(1-z / \alpha_{k, n}\right)\left(1-z / \beta_{k, n}\right) /\left(1-z / \gamma_{n}\right)^{2}
$$

Concerning the paths of reality for $R_{n}(z)$ we have:

(1) The real axis is a path of reality.

(2) The only points at which two paths of reality can intersect are $z=\alpha_{j, n}, z=\beta_{j, n}(j=1,2, \cdots, n), z=\epsilon_{n}, z=-\lambda_{n} / \delta, z=\infty$.

(3) There is one path of reality intersecting the real axis at each of $z=\alpha_{j, n}, z=\beta_{j, n}, z=\epsilon_{n}$ since each of these points is a branch point of order one.

(4) There are $\lambda_{n}-1$ paths of reality intersecting the real axis at $z=-\lambda_{n} / \delta$.

(5) There are $\lambda_{n}$ paths of reality besides the real axis through $z=\infty$.

(6) As in case 1 , no two $\beta_{j, n}$ can lie on the same path of reality.

(7) No path of reality can pass through any $\beta_{j, n}$, and $z=\epsilon_{n}$ for this would result in the intersection of two paths of reality at some point not included in (2).

(8) $\alpha_{n, n}$ and $\epsilon_{n}$ cannot lie on the same path of reality since the real part of $R_{n}(z)$ is a monotone function as $z$ traces a path from $\epsilon_{n}$ to $\alpha_{n, n}$ along the real axis and from $\alpha_{n, n}$ to $\epsilon_{n}$ along a curve in the upper half-plane.

(9) No $\alpha_{j, n}, j \neq n$, can lie on the same path of reality as $\epsilon_{n}$ for the same reason as in (7).

(10) No $\beta_{j, n}$ or $\alpha_{j, n}$ can lie on the same path of reality as $-\lambda_{n} / \delta$ for the same reason.

(11) As in (8) no path of reality can pass through $\epsilon_{n}$ and $-\lambda_{n} / \delta$.

(12) No path of reality through any $\alpha_{j, n}$ or $\beta_{j, n}$ can pass through 
$z=\infty$, for this would require more than $\lambda_{n}+1$ paths of reality through $z=\infty$ or would contradict one of the statements (1)-(11).

Therefore the paths of reality must consist of $n$ curves $C_{k, n}$, $k=1,2, \cdots, n$, and $\lambda_{n}$ curves $D_{k, n}, k=1,2, \cdots, \lambda_{n}$. The curves $C_{k, n}$ are simple closed curves, nonintersecting and symmetric about the real axis with $C_{j, n}$ intersecting the real axis in $z=\alpha_{j, n}, \beta_{j, n}$. The curves $D_{k, n}$ are simple, nonintersecting, and symmetric about the real axis with $D_{1, n}$ passing through $z=\epsilon_{n}$ and $z=\infty$, and $D_{k, n}, k=2$, $3, \cdots, \lambda_{n}$, passing through $z=-\lambda_{n} / \delta$ and $z=\infty$.

As $n \rightarrow \infty$ the point $z=\epsilon_{n}$ and the curves $D_{k, n}$ disappear from consideration, for if $D_{k, n}$ had points interior to $|z|<R<\infty$ for infinitely many $n$, there would be points of $C_{j, n}$ interior to $|z|<R$ for all $j \leqq n$. None of these intersect and in the limit there would be some point $z=z_{0},\left|z_{0}\right|<R,\left|z_{0}-\gamma_{k}\right| \geqq \rho>0$ for all $k$, such that in the neighborhood of $z_{0}$ there would be segments of infinitely many nonintersecting paths of reality of $f(z)$, which is impossible since $f(z)$ is meromorphic in $|z|<\infty$ and hence holomorphic at $z=z_{0}$. So the paths of reality of $f(z)$ in case II are the same as in case $I$, and by the same argument we conclude that if $\delta<0$, the surface of the inverse of $w=f(z)$ is a surface of the first class, which completes the proof of Theorem III.

9. Partial converse of Theorem II. By obvious extensions of the methods used in paragraph 8 we can prove

Theorem IV. Let $w=f(z)$ be meromorphic in $0<|z|<\infty$ with $f(1)$ $=0$ and $f^{\prime}(1)>0$. If $w=f(z)$ has the form

$f(z)=K(z-1) \exp \left(\sigma_{1} z+\sigma_{2} / z\right)$

$$
\prod_{k=1}^{\infty}\left(1-z / c_{k}\right)\left(1-c_{-k} / z\right) /\left(1-z / \gamma_{k}\right)\left(1-\gamma_{-k} / z\right)
$$

where $K$ is real, $\sigma_{1}, \sigma_{2}$ are real and nonpositive, and

$$
\begin{aligned}
& -\infty<\gamma_{k+1}<\gamma_{k}<\gamma_{-k}<\gamma_{-k-1}<0<c_{-k-1}<c_{-k}<1<c_{k}<c_{k+1} \\
& (k=1,2,3, \cdots) \text {, } \\
& \sum_{k=1}^{\infty} 1 / c_{k}<\infty, \quad \sum_{k=1}^{\infty} c_{-k}<\infty, \\
& \sum_{k=1}^{\infty} 1 /\left|\gamma_{k}\right|<\infty, \quad \sum_{k=1}^{\infty}\left|\gamma_{-k}\right|<\infty,
\end{aligned}
$$

then the Riemann surface of the inverse of $w=f(z)$ is a surface of the class described in $\$ 3$. 


\section{ReferenCes}

1. L. Bieberbach, Lehrbsch der Funktionentheorie, 2d ed., vol. 2, Leipzig, 1931

2. C. Caratheodory, Untersuchen wber die konformen Abbildungen von festen und veranderlichen Gebieten, Math. Ann. vol. 72 (1912).

3. - Conformal representation, Cambridge Tracts, no. 28, 1932.

4. L. R. Ford, Automorphic functions, New York, 1929.

5. A. Hurwitz, Über die Nullstellen der Bessel'schen Funktion, Math. Ann. vol. 33 (1889).

6. W. A. Hurwitz and R. Courant, Funktionentheorie, Berlin, 1929.

7. F. Iversen, Recherches sur les fonctions inverses des fonctions meromorphes, Thesis, Helsingfors, 1914.

8. G. R. MacLane, Concerning the uniformization of certain Riemann surfaces allied to the inverse cosine and inverse gamma surfaces, Trans. Amer. Math. Soc. vol. 62 (1947).

9. R. Nevanlinna, Eindeutige analytische Funktionen, Berlin, 1936.

10. B. L. van der Waerden, Topologie und Uniformisierung der Riemann'schen Flachen, Berichte der Sachsischen Akademie zu Leipzig, Math-Phys. Klasse, vol. 93, 8.XII, 1941.

RICE INSTITUTE AND

Florida State University 